\title{
Quality Metrics for Mixed-Signal Indirect Testing
}

\author{
Álvaro Gómez-Pau, Luz Balado, Joan Figueras \\ Departament d'Enginyeria Electrònica, Universitat Politècnica de Catalunya \\ Av. Diagonal 647, 9th floor, 08028 Barcelona (Spain) \\ \{alvaro.gomez-pau, luz.m.balado, joan.figueras\}@upc.edu
}

\begin{abstract}
Analog and mixed-signal circuit testing is a challenging task demanding large amounts of resources. In order to battle against this drawback, alternate testing has been established as an efficient way of testing analog and M-S circuits by using indirect measures instead of the classic specification based testing. In this work we propose the use of Kendall's Tau rank correlation coefficient for rating the suitability of a set of candidate indirect measures to be used in mixed-signal testing. Such criterion is shown to be adequate since it allows to avoid or minimize information redundancy in the measures set. As a proof of concept, a 4th order band-pass Butterworth filter has been simulated under the presence of process variations. The circuit has been tested using a subset of measures selected according to minimum Kendall's Tau coefficient. Analog test efficiency metrics are reported showing test misclassification rate is among the best $15 \%$ possible, therefore validating the proposal.
\end{abstract}

Index Terms-Mixed-Signal Test, Analog Test, Alternate Test, Indirect Measurements, Alternate Feature Selection, Signature Selection, Optimum Measures Selection, Quadtrees, Octrees, Analog Filter

\section{INTRODUCTION}

Testing analog and mixed-signal circuits is a challenging task due to the limitations of current analog automatic test equipment and the partial availability of systematic procedures. This fact causes a significant increase of the incurred costs which are reflected in the final product [1]. Alternate testing methodologies are widely used in contrast to the classic specification based testing and have been presented as a suitable and low cost solution for analog and M-S circuits [2]-[4].

Alternate testing strategies require the selection of a set of easy to measure parameters to be used as indirect measures. To that purpose, many options exist, some of them entirely relying on designer's expertise and experience. Some authors have proposed the use of the sensitivity matrix between circuit's functional specifications and indirect measurements with the goal of maximizing its rank [5]. This allows the avoidance of redundant information. Statistical methods have been also proposed, most of them relying on correlations and regressions techniques between the set of functional specifications and the set of indirect measurements. For instance, in [6], the authors use the Brownian distance correlation together with a greedy algorithm in order to select a meaningful subset of measures adequate for analog/RF circuits testing.

In this work, the use of Kendall's Tau rank correlation coefficient [7] is proposed for rating the suitability of a set of candidate measures to be used in alternate testing.
The underlying concept is to choose the minimum number of measures while avoiding or minimizing the redundancy among them. The method has been applied to test a bandpass Butterworth filter with successful results.

The work is organized as follows. Section II discusses the attributes to be satisfied by an arbitrary set of measures to be used in alternate mixed-signal testing. Kendall's Tau rank correlation coefficient is introduced as a measure of redundancy among all the possible measures. In section III, the case study circuit and the set of candidate test measures are presented. A 4th order band-pass Butterworth filter excited with a multitone signal is used to validate the proposal. Section IV reports some Monte Carlo simulation results. The previously discussed criterion for selecting a reliable set of indirect measures is applied and validated by computing test misclassifications using octrees to encode the acceptance/rejection regions. Finally, section $\mathrm{V}$ summarizes the results and concludes the work.

\section{Indirect MEASURES IN MiXeD-Signal Circuits Testing}

The procedure of testing analog and mixed-signal circuits can be formalized as the classification of any candidate circuit into pass/fail clusters. Such classification can be performed according to a set of parameters of different nature. The most common space for testing is the functional specifications space in which the specifications based testing technique takes place. In this space is where the design goals are directly tested.

Process variations can make the circuit not to be accomplishing the whole set of functional specifications. This is because CUT components (transistors, capacitors, resistors,...) have varied their nominal value inducing the circuit not to be within specifications and therefore failing the test. In this work we will be referring this space as the components space.

Specification based testing can be difficult and time consuming. To overcome these drawbacks, indirect testing methods have been widely adopted as a successful solution. They are based on the measurement of different parameters and use them to perform the test, therefore validating the functional specifications. In this work, the term measure space will be used to identify such space. From now on, the mapping of the pass region in the measure space will be referred as the acceptance region and the fail region as rejection region. The separation between these two regions is the test decision boundary. 
When a candidate set of indirect measurements is proposed to be used for testing, two main properties are desirable for such set. (1) The measures need to reflect circuit's functional specifications variability in order to allow the test to be performed efficiently. (2) An optimum set of measures should not be redundant to avoid incurring in extra costs. Last condition may be relaxed if the objective is to improve test robustness against noisy measures.

In this work, the use of Kendall's Tau rank correlation coefficient [7], [8] is proposed for rating the suitability of a set of candidate measures to be used in alternate testing. Let $\left(x_{1}, y_{1}\right),\left(x_{2}, y_{2}\right), \ldots,\left(x_{n}, y_{n}\right)$ be a set of bivariate data. Given two bivariate data points, $P_{i}=\left(x_{i}, y_{i}\right)$ and $P_{j}=\left(x_{j}, y_{j}\right)$, they are said to be concordant when $x_{i}<x_{j}$ and $y_{i}<y_{j}$ or $x_{i}>x_{j}$ and $y_{i}>y_{j}$, i.e. when components of the difference point $P_{i}-P_{j}$ have the same sign. If they present different sign, the pairs are said to be discordant. Under such definitions, Kendall's Tau rank correlation coefficient [7] is defined as,

$$
\tau=\frac{(\# \text { concordant })-(\# \text { discordant })}{\frac{1}{2} n(n-1)}
$$

Note that $-1 \leq \tau \leq 1$ since the denominator corresponds to the total number of possible pairs given $n$ bivariate data points. Kendall's Tau is a suitable indicator of correlation of any nature since it does not require the data to be linear to present high correlation values. This feature is remarkably desired since in general, the mapping between functional specifications space and indirect measures space is highly nonlinear.

In this work, Kendall's Tau correlation coefficient is used to rate the most adequate measures with the aim of reducing redundant information provided by the selected measurements. In the next section, a continuous time 4th order band-pass filter is presented and the set of indirect measures to be used is defined.

\section{CASe Study: \\ BAND-PASS BUTTERWORTH FILTER}

\section{A. Nominal Circuit}

In order to validate the proposal, a continuous time 4th order band-pass Butterworth filter has been designed and studied under the presence of statistical variability. The nominal filter is characterized by the following transfer function,

$$
H(s)=\frac{G \frac{1}{Q^{2}} \frac{s^{2}}{\omega_{0}^{2}}}{\frac{s^{4}}{\omega_{0}^{4}}+\frac{\sqrt{2}}{Q} \frac{s^{3}}{\omega_{0}^{3}}+\left(2+\frac{1}{Q^{2}}\right) \frac{s^{2}}{\omega_{0}^{2}}+\frac{\sqrt{2}}{Q} \frac{s}{\omega_{0}}+1}
$$

Being $\omega_{0}$ the characteristic angular frequency, $Q$ the quality factor which determines its bandwidth as $f_{2}-f_{1}=f_{0} / Q$ and $G$ the gain in the band-pass. Corner frequencies $f_{1}$ and $f_{2}$ correspond to those frequencies in which the gain is attenuated $3 \mathrm{~dB}$. They can be determined as $f_{0} / F$ and $f_{0} F$ respectively, where $F$ is a dimensionless factor depending on the quality factor, $F=\left(1 / Q+\sqrt{4+1 / Q^{2}}\right) / 2$.

Fig. 1 shows the signal flow graph description of the nominal transfer function, where coefficients $a, b$ and $c$ relate to filter's functional specifications in this way, $a=\sqrt{2} / Q$, $b=2+1 / Q^{2}$ and $c=G / Q^{2}$.

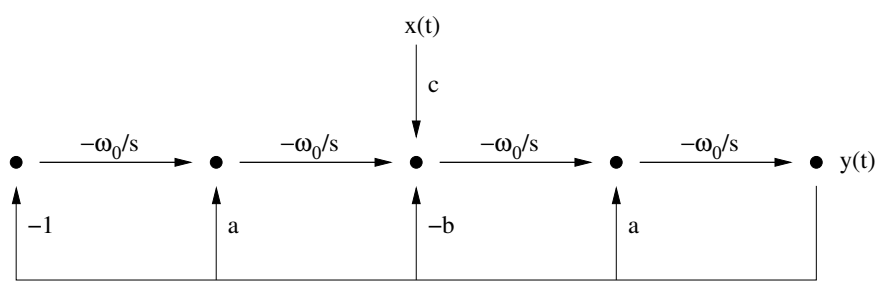

Fig. 1. Signal flow graph of the 4th order Butterworth filter realized with inverting integrators. Its implementation with operational amplifiers is straightforward.

Previous signal flow graph description can be easily implemented in hardware using inverting integrators and a feedback network as the schematic in Fig. 2 depicts [9]. In this case, 5 operational amplifiers and 14 passive components (4 capacitors and 10 resistors) were used.

Passive components $R$ and $C$ determine filter's characteristic frequency as $f_{0}=1 /(2 \pi R C)$. In this work the following component values have been chosen for the nominal filter, $R=1 \mathrm{k} \Omega$ and $C=1.6 \mathrm{nF}$. This selection fixes filter's characteristic frequency at about $f_{0}=100 \mathrm{kHz}$ while the quality factor has been established to $Q=2$ and the gain set to $G=1 \mathrm{~V} / \mathrm{V}$.

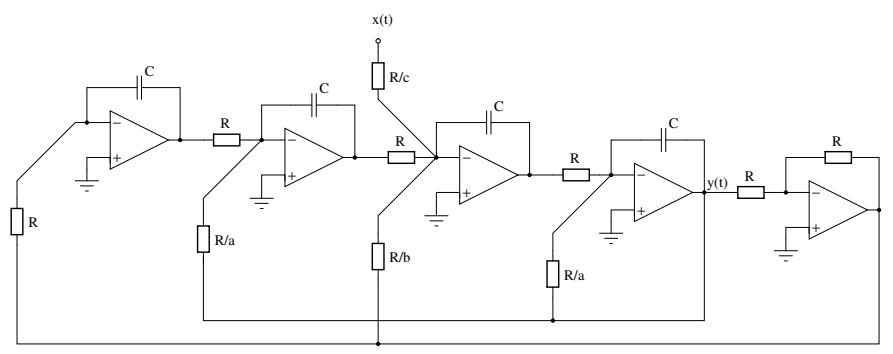

Fig. 2. Schematic of the 4th order band-pass Butterworth filter implemented using passive components and operational amplifiers. The chosen implementation topology corresponds to the so called feedforward form [10].

\section{B. Measures Definition}

In this work, we will use the input/output Lissajous composition method [11] by applying a 3 tone input stimulus to the filter. Such compositions can be considered as an analog signature due to their sensitivity to parametric defects. Lissajous based testing technique composes two DUT signals and uses such information for testing purposes. For instance, Fig. 3 shows the output of the filter depicted in Fig. 2 when it is excited using 3 tone stimulus. The applied tones are tuned at frequencies $f_{0} / 2, f_{0}$ and $2 f_{0}$.

Considering a reference and a deviated Lissajous trace (green and red traces), the measures are defined as the set 


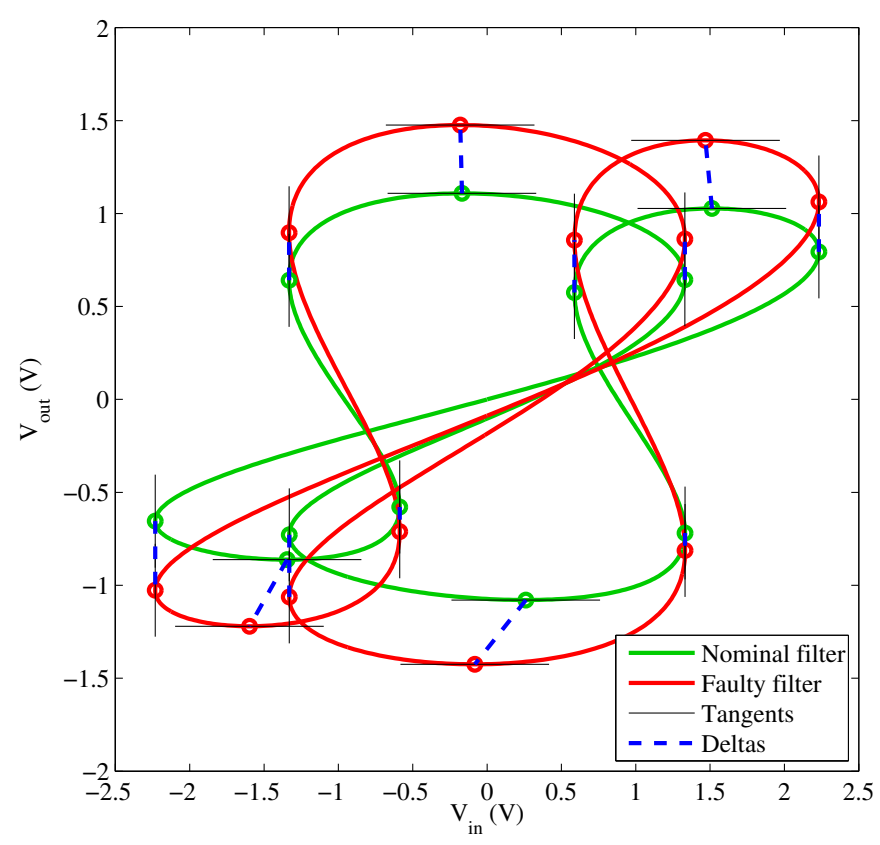

Fig. 3. Input-output composition when a 3 tone signal is applied to the filter sketched in Fig. 2. Green trace corresponds to the nominal filter response while the red trace corresponds to a filter out of specifications.

of $\left(\Delta x_{i}, \Delta y_{i}\right)$ displacements of the shifted curve with respect to the nominal one. The points taken in consideration are the tangency points of vertical and horizontal tangent lines to the Lissajous composition as Fig. 3 depicts. The selection of these points allows an easy and systematic approach facilitating further data processing while keeping Lissajous signature information [12].

\section{Simulation Results}

\section{A. Monte Carlo Simulations}

With the purpose of obtaining a representative sample of circuits population, 5000 Monte Carlo simulations have been carried out considering passive components values distribute according to independent normal distributions. Different $3 \sigma$ spreads have been considered for resistors and capacitors being a $3 \%$ and $5 \%$ of their nominal value respectively. Table I summarizes filter's specifications as well as the lower and upper test limits for each functional specification.

TABLE I

ButTerworth FiLter FunCtional SPECIFICATIONS

\begin{tabular}{|l||l|r|c|c|}
\hline Specification & Symbol & Nominal & Lower & Upper \\
\hline \hline Characteristic freq & $f_{0}(\mathrm{kHz})$ & $100 \pm 2 \%$ & 98 & 102 \\
\hline Band-pass gain & $G(\mathrm{~V} / \mathrm{V})$ & $1 \pm 25 \%$ & 0.75 & 1.25 \\
\hline Quality factor & $Q(-)$ & $2 \pm 30 \%$ & 1.4 & 2.6 \\
\hline
\end{tabular}

In general, given a transfer function affected by component deviations, it is not possible to compare its coefficients against the ones in the nominal transfer function with the aim of deriving its specifications. Because of that, the following rules have been followed to explicitly measure every circuit performances:

- Characteristic frequency is the frequency at which transfer function phase is zero.

- Band-pass gain is the gain at the previously defined characteristic frequency.

- Quality factor is computed by measuring corner frequencies first. Such frequencies correspond to those at which the band-pass gain has been attenuated $3 \mathrm{~dB}$.

The resulting Bode diagrams of the 5000 Monte Carlo filters can be seen in Fig. 4. Green traces correspond to circuits fullfilling the specifications listed in Table I while red traces correspond to circuits violating at least one specification. The thick black trace correspond to the nominal transfer function in Equation (2).
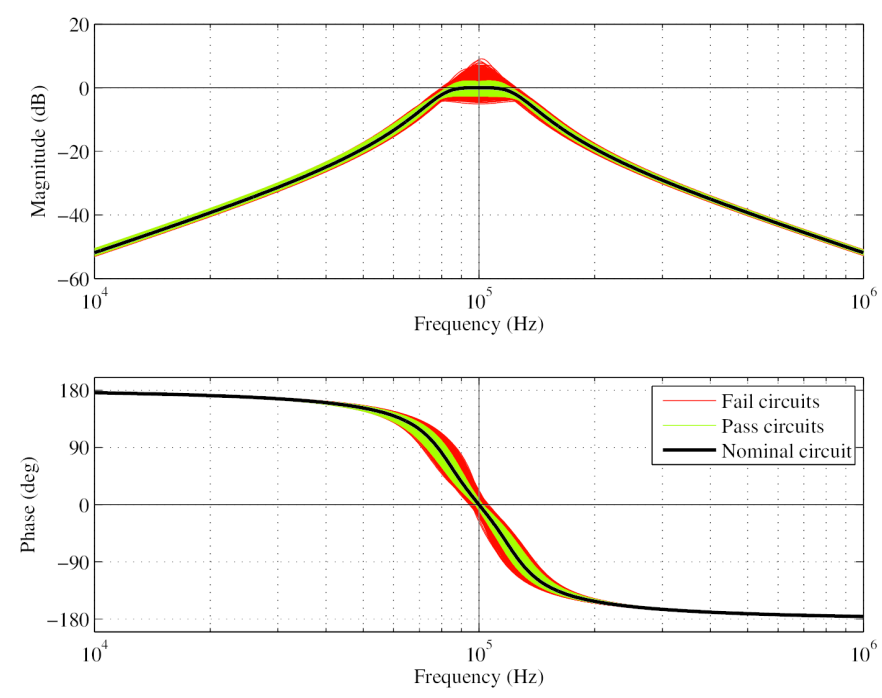

Fig. 4. Bode diagrams obtained by Monte Carlo simulation. Green traces correspond to circuits satisfying all the specifications listed in Table I while red traces correspond to circuits violating, at least, one specification.

Functional specifications histograms and the test acceptance limits of the circuits depicted in Fig. 4 can be seen in Fig. 5. Characteristic frequency can be considered normally distributed according to Anderson-Darling normality test while pass-band gain and quality factor distributions clearly present a nonzero skewness parameter.

The study of possible correlations between functional specifications is desirable in order to find out how many independent variables are involved. Fig. 6 shows the scatter plots of filter's functional specifications, $f_{0}, G$ and $Q$. As can observed, band-pass gain and quality factors are strongly correlated presenting a Pearson linear correlation coefficient of 0.9938 . This fact suggests the total number of independent variables is 2 and therefore only a pair of indirect measures will be required in order to successfully encode the acceptance and rejection regions. 

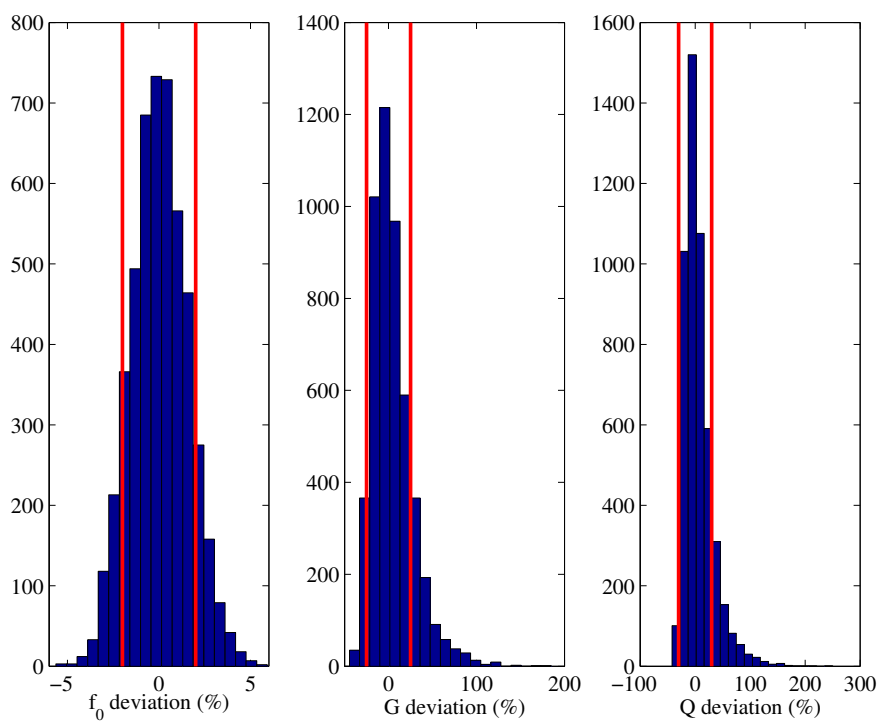

Fig. 5. Histograms of the measured specifications for the set of 5000 Monte Carlo circuits and their test limits. Histograms represent, from left to right characteristic frequency $\left(f_{0}\right)$, pass-band gain $(G)$ and quality factor $(Q)$.

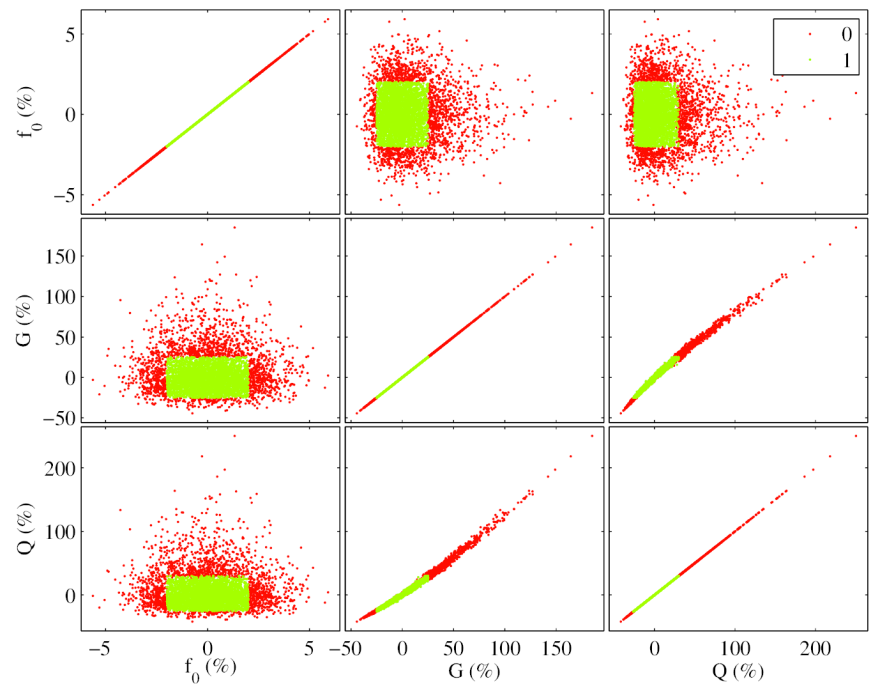

Fig. 6. Scatter plots highlighting Monte Carlo circuits specifications correlations. As can be observed, functional specifications $G$ and $Q$ are strongly correlated with a linear correlation coefficient of 0.9938 .

\section{B. Indirect Measures Selection}

Fig. 7 shows the correlation between filter's functional specifications and the set of 16 indirect measures introduced in section III. Correlations are studied using different indicators, namely, Pearson's linear correlation coefficient, Kendall's Tau rank correlation coefficient [7] and distance correlation [6], [13]. As can be appreciated, all of them seem to be consistent. Pearson's coefficient and distance correlation present a stronger similarity between them than Kendall's Tau, although all 3 show the same tendency.

Section II introduced the need for the indirect measures to reflect the whole variability of the functional specification space. In this case, it is clear they are related, although the highest correlated measure does not necessarily need to be the first to be included in the measures subset since in Fig. 7 only one-to-one correlations are represented.
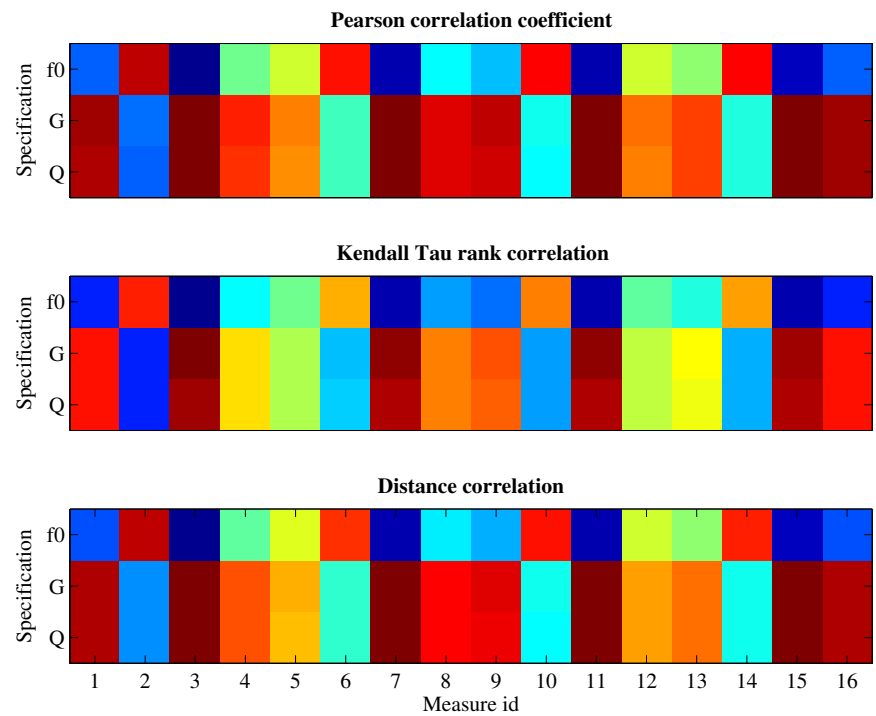

Fig. 7. Different correlation indicators, Pearson coefficient, Kendall's Tau and distance correlation, between filter's functional specifications and the candidate indirect measures. Dark blue indicates no correlation while dark red indicates maximum correlation.

Fig. 6 shows that $f_{0}$ and $Q$ are strongly correlated what allows us to assume 2 effective functional specifications. Because of that, only 2 indirect measures need to be selected since, with certain level of ambiguity, they should be sufficient to perform the test. This fact has been verified by principal component analysis (PCA) method over the set of candidate measures. It reported that with only 2 principal components, the $99.2 \%$ of variability is explained.

In section II it was also mentioned the set of measures need to be independent, i.e. redundancy must be avoided among the selected subset of indirect measures. Because of that, Kendall's Tau rank correlation coefficient is proposed to decide which candidate measures are selected for the testing procedure itself. Table II reports Kendall's Tau correlation coefficient for the set of 16 candidate measures. Fig. 8 reports the same information in colored format.

Then, the pair to be selected as an indirect measure should be the one presenting the minimum Kendall's Tau coefficient. In this case the minimum correlation value is $4.6633 \times 10^{-4}$ corresponding to measure pair (M08,M10). This selection will allow to encode the acceptance and rejection regions using the most independent pair among the 16 possible candidates, i.e. the redundancy in the indirect measures is minimized and therefore test efficiency metrics should improve.

\section{Test Application Results}

In order to validate the proposal, the acceptance and rejection regions using all possible measures pairs have been 
TABLE II

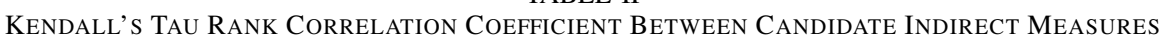

\begin{tabular}{|l||c|c|c|c|c|c|c|c|c|c|c|c|c|c|c|}
\hline & M02 & M03 & M04 & M05 & M06 & M07 & M08 & M09 & M10 & M11 & M12 & M13 & M14 & M15 & M16 \\
\hline \hline M01 & 0.34 & 0.81 & 0.47 & 0.70 & 0.15 & 0.89 & 0.56 & 0.93 & 0.45 & 0.78 & 0.38 & 0.77 & 0.13 & 0.90 & 0.66 \\
\hline M02 & & 0.15 & 0.20 & 0.64 & 0.51 & 0.23 & 0.10 & 0.41 & 0.89 & 0.12 & 0.28 & 0.57 & 0.53 & 0.24 & 0.00 \\
\hline M03 & & & 0.66 & 0.51 & 0.34 & 0.91 & 0.75 & 0.74 & 0.25 & 0.97 & 0.57 & 0.57 & 0.32 & 0.91 & 0.85 \\
\hline M04 & & & & 0.17 & 0.68 & 0.57 & 0.90 & 0.39 & 0.09 & 0.69 & 0.91 & 0.23 & 0.66 & 0.57 & 0.80 \\
\hline M05 & & & & & 0.15 & 0.59 & 0.26 & 0.77 & 0.74 & 0.48 & 0.08 & 0.92 & 0.17 & 0.60 & 0.36 \\
\hline M06 & & & & & & 0.26 & 0.60 & 0.08 & 0.40 & 0.37 & 0.77 & 0.08 & 0.98 & 0.25 & 0.49 \\
\hline M07 & & & & & & & 0.66 & 0.82 & 0.34 & 0.88 & 0.48 & 0.66 & 0.24 & 0.97 & 0.77 \\
\hline M08 & & & & & & & & 0.48 & 0.00 & 0.77 & 0.82 & 0.32 & 0.58 & 0.65 & 0.89 \\
\hline M09 & & & & & & & & & 0.52 & 0.71 & 0.30 & 0.84 & 0.06 & 0.83 & 0.59 \\
\hline M10 & & & & & & & & & & 0.22 & 0.18 & 0.68 & 0.42 & 0.35 & 0.11 \\
\hline M11 & & & & & & & & & & & 0.60 & 0.55 & 0.35 & 0.88 & 0.88 \\
\hline M12 & & & & & & & & & & & & 0.14 & 0.75 & 0.48 & 0.71 \\
\hline M13 & & & & & & & & & & & & & 0.10 & 0.67 & 0.43 \\
\hline M14 & & & & & & & & & & & & & & 0.23 & 0.47 \\
\hline M15 & & & & & & & & & & & & & & & 0.76 \\
\hline
\end{tabular}

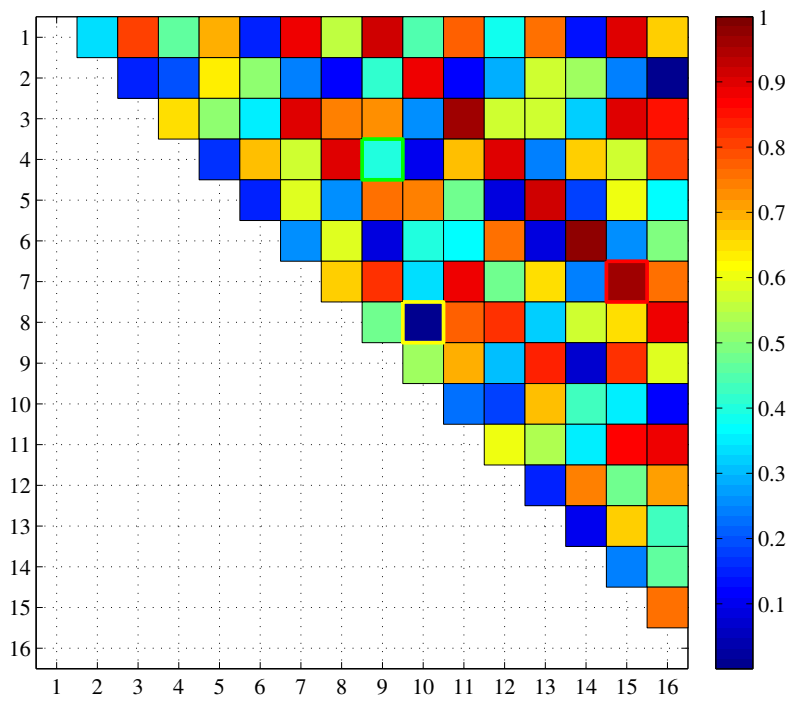

Fig. 8. Colored Kendall's Tau matrix with numerical values shown in Table II Minimum value of correlation $\left(4.6633 \times 10^{-4}\right)$ corresponds to measures M08 and M10. It has been highlighted with a yellow border.

encoded using octrees [14], [15]. Each octree has been tested with a set of $50 \times 10^{3}$ circuits and the number of misclassified circuits evaluated, i.e. test escapes and test yield loss. Fig. 9 shows the resulting octree for the measure pair (M08,M10).

Table III shows the percentage of misclassified circuits using the indicated pairs. As can be observed, maximum value corresponds to a $23.66 \%$ of misclassified circuits using measure pair (M07,M15). A quick check in Table II and Fig. 8 confirms such pair presents one of the maximum values of Kendall's Tau correlation. On the contrary, the minimum percentage of misclassified circuits is for pair (M04,M09) with $1.99 \%$. For the case of the selected pair (M08,M10), the rate of misclassified circuits is $2.55 \%$ which is located in percentile $15 \%$ among all the obtained misclassification rates.

There exists a remarkable similitude in the patterns appreciated in Fig. 8 and Fig. 10. Those pairs presenting highest

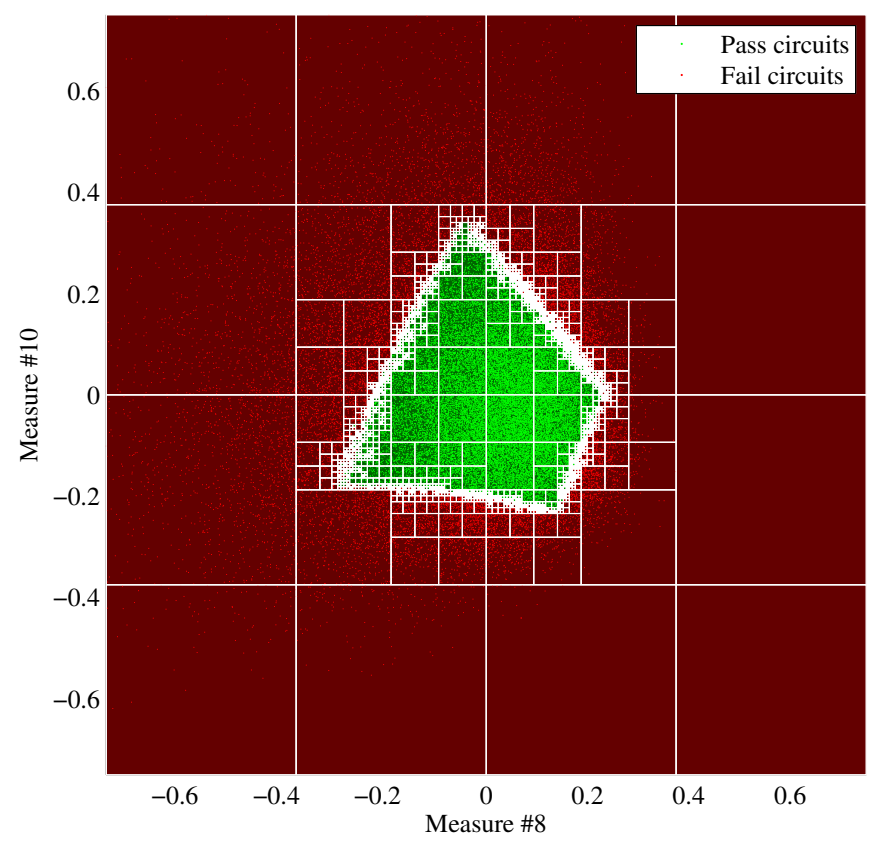

Fig. 9. Resulting octree encoding the acceptance and rejection regions when measures M08 and M10 are selected for testing. The octree evaluation allows the test decision. Higher octree levels concentrate near the test decision boundary [15].

Kendall's Tau values correspond to those pairs exhibiting the largest test misclassification rates. A noticeable example is the diagonal departing from pair (M01,M09) and going down to pair (M08,M16) in which warm colors clearly prevail.

\section{CONCLUSIONS}

A criterion for rating the suitability of indirect measures to be used in alternate testing of mixed-signal circuits has been proposed. The method relies on the use of Kendall's Tau rank correlation coefficient as indicator. The presented criterion considers those candidate measures reporting the minimum Kendall's Tau coefficient, therefore avoiding or minimizing 
TABLE III

Octree Test Results Showing the Percentage of Misclassified Circuits for Every Measure Pair

\begin{tabular}{|l||r|r|r|r|r|r|r|r|r|r|r|r|r|r|r|}
\hline & M02 & M03 & M04 & M05 & M06 & M07 & M08 & M09 & M10 & M11 & M12 & M13 & M14 & M15 & M16 \\
\hline \hline M01 & 2.45 & 3.66 & 2.48 & 4.34 & 3.15 & 7.34 & 2.66 & 8.41 & 2.84 & 3.91 & 2.34 & 5.60 & 2.98 & 9.75 & 3.20 \\
\hline M02 & & 2.59 & 2.55 & 5.60 & 4.67 & 2.89 & 2.98 & 3.09 & 10.22 & 2.67 & 3.13 & 5.13 & 4.66 & 2.94 & 2.55 \\
\hline M03 & & & 2.53 & 2.70 & 3.69 & 8.84 & 4.38 & 3.02 & 2.42 & 8.44 & 2.52 & 2.74 & 3.21 & 5.85 & 5.79 \\
\hline M04 & & & & 2.75 & 6.20 & 3.45 & 12.89 & 1.99 & 2.25 & 2.91 & 7.90 & 2.42 & 5.49 & 2.27 & 3.88 \\
\hline M05 & & & & & 3.66 & 3.39 & 3.13 & 6.37 & 7.75 & 2.69 & 2.77 & 16.71 & 3.26 & 4.17 & 3.10 \\
\hline M06 & & & & & & 3.65 & 4.29 & 2.97 & 3.62 & 4.00 & 8.90 & 3.36 & 13.58 & 3.09 & 3.74 \\
\hline M07 & & & & & & & 2.98 & 6.90 & 3.35 & 7.23 & 2.83 & 5.49 & 3.39 & 23.66 & 5.23 \\
\hline M08 & & & & & & & & 2.93 & 2.55 & 5.69 & 5.83 & 3.23 & 4.10 & 3.77 & 10.36 \\
\hline M09 & & & & & & & & & 2.66 & 3.06 & 2.23 & 5.81 & 2.75 & 3.46 & 2.15 \\
\hline M10 & & & & & & & & & & 2.53 & 2.29 & 4.47 & 3.37 & 2.49 & 2.43 \\
\hline M11 & & & & & & & & & & & 2.93 & 2.80 & 3.60 & 5.14 & 8.34 \\
\hline M12 & & & & & & & & & & & & 2.67 & 7.71 & 2.59 & 4.44 \\
\hline M13 & & & & & & & & & & & & & 3.19 & 3.28 & 2.94 \\
\hline M14 & & & & & & & & & & & & & & 2.86 & 3.43 \\
\hline M15 & & & & & & & & & & & & & & & 2.75 \\
\hline
\end{tabular}

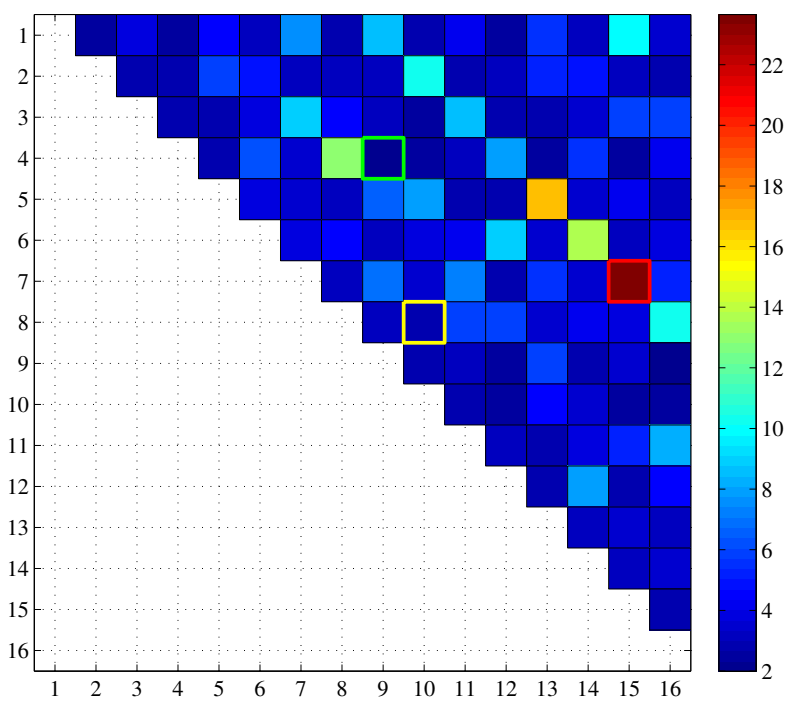

Fig. 10. Percentage of misclassified circuits (test escapes and test yield loss) with numerical values shown in Table III. The minimum Kendall's Tau measure pair is highlighted in yellow. Pairs giving the lowest and highest misclassification rate are highlighted in green and red respectively.

redundancy in the information provided by each measure what immediately translates in better test results. The presented method can be naturally extended to arbitrary dimension spaces.

The proposed method has been applied to select, among a candidate set, a subset of indirect measures for testing a band-pass Butterworth filter under the presence of process variations. The resulting selected measures using the proposed criterion have been used to encode the measure space using octrees. Test misclassification for such measures is among the best $15 \%$ possible pairs, therefore validating the proposal.

\section{ACKNOWLEDGMENTS}

This work has been partially supported by the Spanish Ministry of Economics and Competitiveness (project reference is TEC2010-18384) and European Union FEDER funds.

\section{REFERENCES}

[1] L. Milor, "A tutorial introduction to research on analog and mixedsignal circuit testing," Circuits and Systems II: Analog and Digital Signal Processing, IEEE Transactions on, vol. 45, no. 10, pp. 1389-1407, 1998.

[2] A. Chatterjee and P. Variyam, "Enhancing test effectiveness for analog circuits using syntesized measurements," in VLSI Test Symposium, 1998, pp. $132-137$

[3] S. Devarakond, S. Sen, S. Bhattacharya, and A. Chatterjee, "Concurrent device/specification cause-effect monitoring for yield diagnosis using alternate diagnostic signatures," Design Test of Computers, IEEE, vol. 29, no. 1, pp. 48-58, 2012.

[4] H.-G. Stratigopoulos and Y. Makris, "Error moderation in low-cost machine-learning-based analog/rf testing," Computer-Aided Design of Integrated Circuits and Systems, IEEE Transactions on, vol. 27, no. 2, pp. 339-351, 2008.

[5] A. Chatterjee and S. Cherubal, "Method for diagnosing process parameter variations from measurements in analog circuits," June 2002, uS Patent App. 09/838, 404.

[6] M. Barragan and G. Leger, "Efficient Selection of Signatures for Analog/RF Alternate Test," in Test Symposium (ETS), 2013 18th IEEE European, May 2013, pp. 1-6.

[7] M. G. Kendall, "A New Measure of Rank Correlation," Biometrika, vol. 30, no. 1/2, pp. 81-93, June 1938.

[8] A. Bounceur, S. Mir, and H. Stratigopoulos, "Estimation of Analog Parametric Test Metrics Using Copulas," Computer-Aided Design of Integrated Circuits and Systems, IEEE Transactions on, vol. 30, no. 9, pp. 1400-1410, September 2011.

[9] P. Mohan, VLSI Analog Filters: Active $R C$, OTA-C, and SC, ser Modeling and Simulation in Science, Engineering and Technology. Birkhäuser, 2012.

[10] H. G. Dimopoulos, Analog Electronic Filters. Theory, Design and Synthesis. New York: Springer, 2012.

[11] L. Balado, E. Lupon, J. Figueras, M. Roca, E. Isern, and R. Picos, "Verifying Functional Specifications by Regression Techniques on Lissajous Test Signatures," IEEE Transactions on Circuits and Systems I: Regular Papers, vol. 56, pp. 754-762, April 2009, issue 4.

[12] A. Gómez-Pau, L. Balado, and J. Figueras, "Nondestructive Diagnosis of Mechanical Misalignments in Dual Axis Accelerometers," in Proceedings of Design, Test, Integration \& Packaging of MEMS/MOEMS (DTIP), Barcelona, Spain, April 2013, pp. 229-234.

[13] G. J. Székely, M. L. Rizzo, and N. K. Bakirov, "Measuring and testing dependence by correlation of distances," The Annals of Statistics, vol. 35, no. 6, pp. 2769-2794, December 2007.

[14] D. Meagher, "Octree Encoding: A New Technique for the Representation, Manipulation and Display of Arbitrary 3-D Objects by Computer,' Rensselaer Polytechnic Institute, New York, Tech. Rep., 1980.

[15] A. Gómez-Pau, L. Balado, and J. Figueras, "M-S Test Based on Specification Validation using Octrees in the Measure Space," in Proceedings of IEEE European Test Symposium (ETS), Avignon, France, May 2013, pp. 70-75. 